Cite this: Food Funct., 2014, 5, 755

\title{
Numerical simulation of adsorption and bubble interaction in protein foams using a lattice Boltzmann method
}

\begin{abstract}
Daniela Anderl, ${ }^{\text {a }}$ Martin Bauer, ${ }^{\mathrm{b}}$ Cornelia Rauh, ${ }^{\mathrm{a}}$ Ulrich Rüde ${ }^{\mathrm{b}}$ and Antonio Delgado ${ }^{\mathrm{a}}$
The adsorption process and the resulting dynamic surface tension in the context of protein foams were studied. A diffusion-advection equation is solved using a lattice Boltzmann method (LBM) in order to simulate the adsorption of surfactants on a surface. With different adsorption isotherms, different surfactants can be modelled. The advection is driven by a flow field coming from the LBM. The phase transition is implemented with a free surface LBM approach where the liquid-gas two-phase flow is simplified to a single-phase free surface flow by using a volume of fluid approach. Looking at the different time scales for diffusion and advection, which are determined by the diffusion coefficient and the viscosity, respectively, the LBM is limited due to time and space resolution. The rates of protein transport to a surface by diffusion and by advection are investigated which indicate that diffusion is only relevant for modelling long-time studies. For those time ranges and low concentrations, the diffusion of proteins from a bulk to a surface of a droplet is simulated and compared with the literature. As a next step, situations as in protein foams are assumed. High concentrations of proteins, e.g. as in milk, result in a simplified scenario where neither diffusion nor advection is important. This is analysed theoretically which suggests an instantaneous change of surface tension. To examine the stability of foam lamellae, this is used for further simulations. Two bubbles rise close to each other with globally different surface tensions as for pure water and water with proteins. Depending on these surface tensions and the initial distance, the bubbles coalesce faster for high surface tensions and show less secondary motions for lower surface tension. It is concluded that

bubbles in protein foams coalesce only at shorter distances than in pure water.
\end{abstract}

Received 30th August 2013

Accepted 11th January 2014

DOI: $10.1039 / c 3 f o 60374 a$

www.rsc.org/foodfunction

\section{Introduction}

Protein foams find widespread application in the food industry. Many products such as whipped cream, latte macchiato, milkshakes and ice cream are examples in industry of the importance of food with a high proportion of dispersed gas. In addition to production and processing, the stability of the foam right after the formation of new surfaces or later during transport or storage is also relevant.

Foam stability depends mostly on processes within the liquid phase between neighbouring bubbles, called foam lamellae. At this scale, the lifetime of a lamella depends very strongly on the flow field which arises from the bubble-bubble interaction. The flow field in lamellae develops from gravitational acceleration depending on the position of the lamella relative to it and in addition on effects due to proteins.

Proteins in the solution adsorb due to diffusion and advection to newly created surfaces. The adsorption time depends on the

${ }^{a}$ Friedrich-Alexander University Erlangen-Nuremberg, Chair of Fluid Mechanics, Cauerstr. 4, Erlangen, Germany.E-mail: daniela.anderl@fau.de

${ }^{b}$ Friedrich-Alexander University Erlangen-Nuremberg, Chair of System Simulation, Cauerstr. 11, Erlangen, Germany concentration of the proteins and on the time the surface was exposed to the solution. Once the proteins have adsorbed onto the surface of the bubble, the surface tension changes. This change in surface tension influences the flow field in the lamellae and therefore the foam stability. The proteins studied in this work were the milk proteins, $\beta$-lactoglobulin (BLG) and $\beta$-casein. ${ }^{1}$

Owing to different time and length scales, the stabilization of foams by proteins is difficult to investigate. As it is a crucial phenomenon to predict foam stability and deformability, numerical simulations are fundamental tools for analysis. With numerical methods, flow fields around and between bubbles and therefore lamellae stability can be analysed at every time and length scale.

A very early approach to investigate the adsorption process by diffusion analytically was reported by Ward and Tordai. ${ }^{2}$ Their equation models the adsorption of a surfactant under the assumptions that the surfactant is non-ionic and the activation energy for adsorption is zero. The adsorption model consists of two processes, namely the diffusive transport of the surfactant to the surface from a solution with uniform bulk concentration and back-diffusion from the surface to the liquid. Since only diffusive aspects are considered, the Ward-Tordai equation can be derived from the diffusion equation. Ward and Tordai ${ }^{2}$ gave 
the solution for a plane surface with a semi-infinite domain. Mysels $^{3}$ formulated the Ward-Tordai equation for spherical surfaces. Li et al. ${ }^{4}$ solved this form of the Ward-Tordai equation numerically by rewriting it in the form of a Volterra equation and using different well-known adsorption isotherms.

By the adsorption isotherm, the surfactant enters the model. Proteins, as the special surfactant considered here, can be modelled by an adsorption isotherm accounting for the change of surface excess of proteins due to folding. ${ }^{5-7}$

With the Ward-Tordai approach, Miller et al. ${ }^{5}$ used the developed adsorption isotherm for proteins ${ }^{6}$ to compute the dynamic surface tension. Yang and $\mathrm{Gu}^{8}$ used a finite element approach to model the adsorption kinetics of simpler surfactants in a pendant drop and compared also with the solution from the Ward-Tordai equation. By contrast, Yang et al. ${ }^{9}$ considered a pendant bubble and additionally also its shape.

Instead of using approximations for special geometries, here the mentioned diffusion equation is used directly and an advection term is added. The resulting diffusion-advection equation is then solved with the LBM. The interface between the liquid and the gas is treated by an extension of the LBM, the free surface LBM. For validation a simple diffusive adsorption problem was chosen, similar to Yang and Gu. ${ }^{8}$

Diffusion LBM is already established ${ }^{\mathbf{1 0}}$ and has already been used to model heat and moisture transfer in cereal foams. ${ }^{\mathbf{1 1 , 1 2}}$ Also, the diffusion-advection equation has been simulated with LBM and applied to physical problems. ${ }^{\mathbf{1 3 , 1 4}}$

Using the LBM to model the adsorption of proteins, difficulties regarding the time scales arise. The diffusion process of proteins in water happens at very large time scales whereas the flow phenomena which determine the advection occur at smaller time scales. This problem is discussed in depth for realistic quantities regarding protein foams. The consequence is that for protein foams and their processing, constant changes in surface tension due to protein adsorption are sufficient to describe the flow field in the lamellae. Those flow fields are analysed regarding the change due to protein adsorption and the stability of the lamellae also under the influence of gravity.

In Section 2, the computational model, LBM for diffusionadvection and for the Navier-Stokes equations and the free surface LBM to handle the interface, is introduced. In addition, a validation scenario with results is given. Section 3 makes the transition to the part where surface tension changes can be assumed instantaneously. Based on this, Section 4 investigates the stability of lamellae by simulating two close, rising bubbles. Section 5 summarizes the work.

\section{Numerical model}

\subsection{Lattice Boltzmann Method (LBM)}

The LBM is based on the discretization of the Boltzmann equation. ${ }^{15,16}$ The Boltzmann equation comes from statistical mechanics and reads with a simplified collision term

$$
\frac{\partial f}{\partial t}+\vec{\xi} \cdot \nabla f=Q(f, f) .
$$

$f(\vec{x}, \vec{\xi}, t)$ is the continuous probability density function that yields the probability of meeting a particle with velocity $\vec{\xi}$ at position $\vec{x}$ at time $t .^{17,18}$

For the collision term, the model from Bhatnagar, Gross and Krook $^{\mathbf{1 9}}$ is chosen for simplicity

$$
Q(f, f)=\frac{1}{\tau}\left(f^{\text {eq }}-f\right)
$$

where the probabilities are relaxed with the relaxation time $\tau$ towards the local equilibrium distribution $f^{\text {eq }}$ given by a Maxwell distribution.

To solve (1) with numerical methods, the velocity space has to be discretized with a certain number of degrees of freedom. This results in a computational lattice. Here the three-dimensional lattice model D3Q19 is used, where D3 denotes three dimensions and Q19 is the number of discrete velocities. ${ }^{20}$ The corresponding discrete particle distribution functions (PDFs) are denoted by

$$
f(\vec{x}, \vec{\xi}, t) \rightarrow f\left(\vec{x}, \vec{e}_{\alpha}, t\right)=f_{\alpha}(\vec{x}, t)
$$

$\alpha$ ranges from 0 to 18 for the $\mathrm{Q} 19$. The discrete lattice velocities are denoted by $\vec{e}_{\alpha}$. Using a Taylor expansion, the Maxwell distribution is discretized and reads

$$
f_{\alpha}^{\mathrm{eq}}=\rho w_{\alpha}\left[1+\frac{\vec{e}_{\alpha} \cdot \vec{u}}{c_{\mathrm{s}}{ }^{2}}+\frac{\left(\vec{e}_{\alpha} \cdot \vec{u}\right)^{2}}{2 c_{\mathrm{s}}{ }^{4}}-\frac{\vec{u} \cdot \vec{u}}{2 c_{\mathrm{s}}{ }^{2}}\right]
$$

where $w_{\alpha}$ are weights according to the lattice model used.

Since the PDFs are only mesoscopic quantities, the macroscopic quantities, also appearing in (4), are obtained by taking the moments of the PDFs. For the present computation, it is sufficient to compute the density $\rho$ and the velocity vector $\vec{u}$ by the zero and first moment:

$$
\begin{gathered}
\rho=\sum_{\alpha} f_{\alpha} \\
\vec{u}=\frac{1}{\rho} \sum_{\alpha} \vec{e}_{\alpha} f_{\alpha}
\end{gathered}
$$

The time and space discretization yields an updating rule for the PDFs which can be split into a collision step (7) and a stream step (8).

$$
\begin{gathered}
\tilde{f}_{\alpha}(\vec{x}, t+\Delta t)=f_{\alpha}(\vec{x}, t)-\frac{1}{\tau_{v}}\left[f_{\alpha}(\vec{x}, t)-f_{\alpha}^{\mathrm{eq}}(\rho, \vec{u})\right] \\
f_{\alpha}\left(\vec{x}+\vec{e}_{\alpha} \Delta t, t+\Delta t\right)=\tilde{f}_{\alpha}(\vec{x}, t+\Delta t)
\end{gathered}
$$

The relaxation time $\tau$ is written with subscript $\nu$ to distinguish it from the relaxation time in Section 2.3. It can be expressed by the lattice viscosity $\nu_{\mathrm{L}}$, which is related to the physical kinematic viscosity as explained in Section 2.3.2 and reads

$$
\tau_{v}=\frac{\nu_{\mathrm{L}}}{c_{\mathrm{s}}^{2}}+\frac{1}{2} \text {. }
$$

The discrete scheme follows an ideal gas equation of state where the pressure is given by

$$
p=\rho c_{\mathrm{s}}^{2},
$$


with the lattice speed of sound $c_{\mathrm{s}}$ as a lattice model-dependent parameter (here, $c_{\mathrm{s}}=1 / \sqrt{3}$ ).

To obtain the Navier-Stokes equations from this mesoscopic lattice Boltzmann equation or from the continuous Boltzmann equation, the Chapman-Enskog analysis has to be applied..$^{15,17,21}$ This analysis shows that the described LBM simulates from a macroscopic view the Navier-Stokes equations.

\subsection{Free surface LBM (FSLBM)}

To model the presence of two phases, e.g. water and air, the FSLBM is used. It is based on the assumption that the simulated liquid-gas flow is completely governed by the heavier phase and the dynamics of the lighter or gas phase can therefore be neglected. Hence the problem is reduced to a single-phase flow with a free boundary. ${ }^{22}$ The heavier phase is simulated with LBM as described in Section 2.1 and termed liquid phase in the following. The boundary at the free surface is treated by a volume of fluid approach. Cells which are between the two phases are denoted as interface cells. A cell is a cubic volume of unit length centred around a lattice node. The interface cells form a closed layer between the gas and the liquid cells. A variable $\varphi$ for every interface cell holds the actual fill level and varies between 0 for gas only and 1 for a cell completely filled with liquid. When the fill level exceeds such a limit, the respective interface cell is transformed into a gas cell (if $\varphi<0$ ) or into a liquid cell (if $\varphi>1$ ).

Unconnected gas regions which arise from this kind of topology changes are tracked with a special bubble model. ${ }^{23-25}$ This model tracks the actual volume of such gas regions and the initial volume from the beginning of the simulation. Those two values yield a bubble pressure which is necessary for the boundary treatment at the free surface. ${ }^{22}$ The other pressure which is important for the boundary treatment is the Laplace pressure (11) coming from surface tension effects.

$$
\Delta p_{\sigma}=2 \sigma \kappa
$$

The curvature $\kappa$, which is a function of place and time, is computed locally for every interface cell from the fill levels of its neighbours. The surface tension $\sigma$ can be defined as a global value for the whole simulation or as a local value depending on the surface excess.

\subsection{Diffusion-advection LBM}

Instead of using approximations as in the Ward-Tordai approach, a diffusion-advection eqn (12) is solved with LBM for all cells in the liquid region.

$$
\frac{\partial c}{\partial t}=\nabla \cdot(D \nabla c)-\nabla \cdot(c \vec{u})
$$

The protein concentration is denoted by $c$. From the hydrodynamic LBM, see Section 2.1, the velocity $\vec{u}$ in the advection term is available. To solve (12) with LBM, an additional set of distribution functions $g_{\alpha}$ with the same lattice discretization as for the hydrodynamics is used. The macroscopic quantity $c$ is obtained from the zero moment of this set of PDFs

$$
c=\sum_{\alpha} g_{\alpha} .
$$

The collision and stream steps are the same as in Section 2.1.

$$
\begin{gathered}
\tilde{g}_{\alpha}(\vec{x}, t+\Delta t)=g_{\alpha}(\vec{x}, t)-\frac{1}{\tau_{\mathrm{D}}}\left[g_{\alpha}(\vec{x}, t)-g_{\alpha}^{\mathrm{eq}}(c, \vec{u})\right] \\
g_{\alpha}\left(\vec{x}+\vec{e}_{\alpha} \Delta t, t+\Delta t\right)=\tilde{g}_{\alpha}(\vec{x}, t+\Delta t)
\end{gathered}
$$

The equilibrium function $g_{\alpha}^{\mathrm{eq}}$ is only an expansion up to firstorder terms

$$
g_{\alpha}^{\mathrm{eq}}=c w_{\alpha}\left[1+\frac{\vec{e}_{\alpha} \cdot \vec{u}}{c_{\mathrm{s}}^{2}}\right]
$$

because only the zero moment is evaluated. Since the diffusion occurs at a different time scale, also the relaxation time $\tau_{\mathrm{D}}$ is different. It is not defined by the lattice viscosity as for the hydrodynamic LBM, but by the diffusion coefficient in lattice units ${ }^{\mathbf{1 0}}$

$$
\tau_{\mathrm{D}}=\frac{D_{\mathrm{L}}}{c_{\mathrm{s}}^{2}}+\frac{1}{2}
$$

The macroscopic velocity $\vec{u}$ is taken from the hydrodynamic LBM.

2.3.1 Boundary conditions at the free surface for adsorption. The crucial point here is how to incorporate the surface model from Section 2.2 into the boundary condition. Following the assumptions of the Ward-Tordai approximation, we use the interface cells as the boundary and prescribe a concentration $c$ there (Fig. 1). This is basically an inhomogeneous Dirichlet boundary since the value for $c$ changes potentially in every time step as the excess changes. The concentration at the interface is assumed to be dependent only on the surface excess $\Gamma$. The relationship between the concentration and the surface excess is modelled by the adsorption isotherm $c(\Gamma)$. Even though the adsorption isotherm is valid for equilibrium and not for an

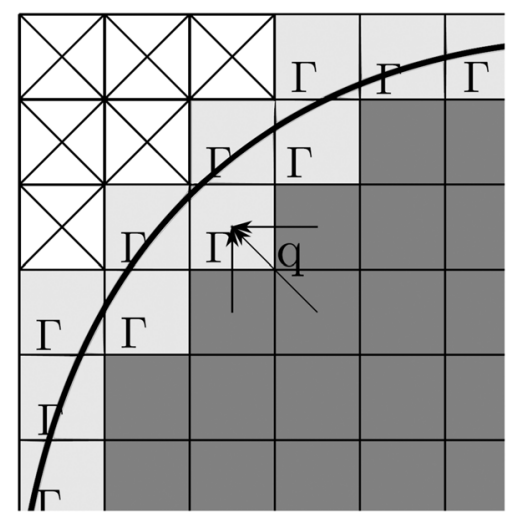

Fig. 1 Schematic view of the boundary situation. The actual boundary is the bold line. The bulk concentration is computed with LBM in all dark grey cells (liquid cells). The crossed cells are gas cells which are not part of the computational domain. The light grey cells, containing the integrated surface excess $I$, are interface cells. 
instantaneous change of surface excess, this is a familiar and reasonable approach., ${ }^{\mathbf{4 , 5}}$

The surface excess is a function of the fluxes over the surface $q=D \frac{\partial c}{\partial n}$ as used in the Ward-Tordai equation. Here also the diffusion coefficient of the proteins in the solution $D$ is of importance. Since the surface is modelled with interface cells (see Section 2.2), the fluxes to and from those cells are tracked as reported by Attar and Körner: ${ }^{26}$

$$
\vec{q}^{\text {gas }}=\underbrace{\sum_{\alpha / 2} g_{\alpha}^{\text {out }}\left(\vec{e}_{\alpha}-\vec{u}\right)}_{\left(\vec{e}_{\alpha}-\vec{u}\right) \cdot \vec{n}<0}+\underbrace{\sum_{\bar{\alpha} / 2} g_{\alpha}^{\text {in }}\left(\vec{e}_{\alpha}-\vec{u}\right)}_{\left(\vec{e}_{\alpha}-\vec{u}\right) \cdot \vec{n} \geq 0}
$$

where $\vec{q}^{\text {gas }}$ denotes the flux coming from the liquid going into the gas. The PDFs are summed according to their direction with respect to the surface normal $\vec{n}$. The first half are the PDFs coming from the liquid and the second half are those coming from the surface going into the liquid cells. For the excess, only the normal portion of the flux is needed, so the final flux which has to be integrated reads

$$
q=D\left(\vec{q}^{\mathrm{gas}} \cdot \vec{n}\right)
$$

The tracked fluxes over time are summed and stored in the interface cells as surface excess $\Gamma$ and therefore influence the boundary concentration via the isotherm. In Fig. 1, the fluxes coming from the adjacent liquid cell to one interface cell are visualized by arrows for one cell.

The explained algorithm is summarized in Algorithm 1 and integrated into the previously explained diffusion-advection LBM.

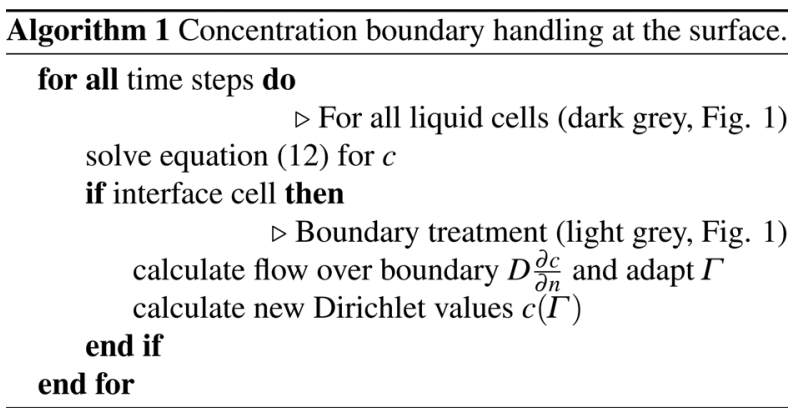

2.3.2 From physical units to lattice units. ${ }^{27}$ As already mentioned, there is a set of variables which is in lattice units. Those lattice variables are used to match the physical quantities to the lattice time and space step in the simulation which are equal to one by definition and dimensionless. To solve a physical problem, these variables have to be computed by choosing an appropriate physical time step $\mathrm{d} t$, physical space step $\mathrm{d} x$ and a relaxation time $\tau$. With $\mathrm{d} x, \mathrm{~d} t$ and $\tau$, the physical variables which have only metre and second in their units can be made dimensionless. Taking as an example the kinematic viscosity $\nu$ which has the SI unit $\mathrm{m}^{2} \mathrm{~s}^{-1}$, the viscosity in lattice units is

$$
\nu_{\mathrm{L}}=\nu \cdot \frac{\mathrm{d} t}{\mathrm{~d} x^{2}} .
$$

All physical parameters have to be scaled respectively to obtain a parameter set in lattice units for the actual simulation.

\subsection{Validation}

2.4.1 Choice of model and simulation set-up. To come from the surface excess to a concentration at the boundary, an adsorption isotherm is needed. There is a model for the adsorption isotherm for proteins which also accounts for the unfolding during the adsorption process. ${ }^{5-7}$ For a validation scenario, a simpler Langmuir adsorption isotherm was chosen ${ }^{8}$

$$
c=\frac{a_{\mathrm{L}} \Gamma}{\Gamma_{\mathrm{m}}-\Gamma} .
$$

To compare the results with those of Yang and $\mathrm{Gu},{ }^{8}$ the constants were chosen equally. The Langmuir adsorption constant is $a_{\mathrm{L}}=5.44 \times 10^{-3} \mathrm{~mol} \mathrm{~m}^{-3}$ and the maximum excess is $\Gamma_{\mathrm{m}}=1.8 \times 10^{-6} \mathrm{~mol} \mathrm{~m}^{-2}$. Using also the same diffusion coefficient $D=5 \times 10^{-10} \mathrm{~m}^{2} \mathrm{~s}^{-1}$, the diffusion is slightly faster than for proteins since the diffusion coefficient for $e . g$. $\beta$-casein is around $0.6 \times 10^{-10} \mathrm{~m}^{2} \mathrm{~s}^{-1}$ according to Miller et al. ${ }^{5}$

The physical scenario of the simulation is a spherical droplet with a radius of $r=1.68 \times 10^{-3} \mathrm{~m}$ and a discretization of 40 cells for the diameter. To model also the diffusive transport to the surface, a centred spherical domain with a radius of $r_{\text {init }}=$ $1 \times 10^{-3} \mathrm{~m}$ within the droplet is set to a constant bulk concentration as sketched in Fig. 2. This models the source of surfactants. The discretization determines $\mathrm{d} x=8.4 \times 10^{-5} \mathrm{~m}$. To choose the time step, the stability criteria for LBM have to be met, which means that the relaxation times have to be larger than $0.5 .^{28,29}$ This is not trivial since there are two different relaxation times for diffusion (17) and hydrodynamics (9). Since the chosen liquid should be water, which has a kinematic viscosity of $\nu=1 \times 10^{-6} \mathrm{~m}^{2} \mathrm{~s}^{-1}$ and in addition the relaxation time should not be too large, to have sufficient accuracy, the relaxation time for the diffusion has to be chosen quite close to the stability border 0.5 . Fixing $\tau_{v}$ yields a lattice viscosity according to Section 2.3.2 which determines the time step to be $\mathrm{d} t=3.528 \times 10^{-3} \mathrm{~s}$. Hence the relaxation time is $\tau_{\mathrm{D}}=0.50225$.

The initial surface excess is zero at the first time step. The whole liquid domain is initially set to the bulk concentration.

2.4.2 Results. The numerical simulations with the previously described set-up show good agreement with Yang and

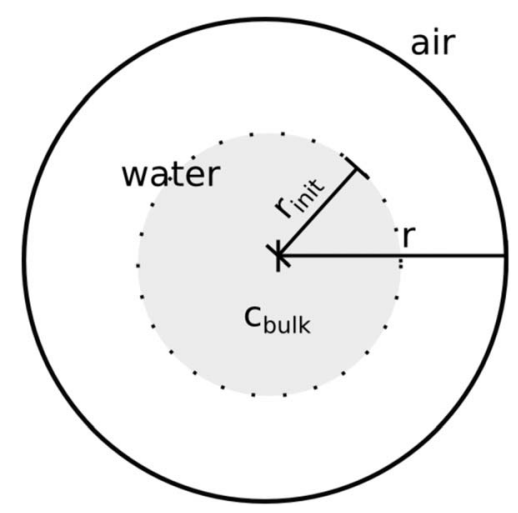

Fig. 2 Schematic two-dimensional view of the simulation set up. The gray area has a constant bulk concentration. The continuous line is the boundary of the liquid domain. 


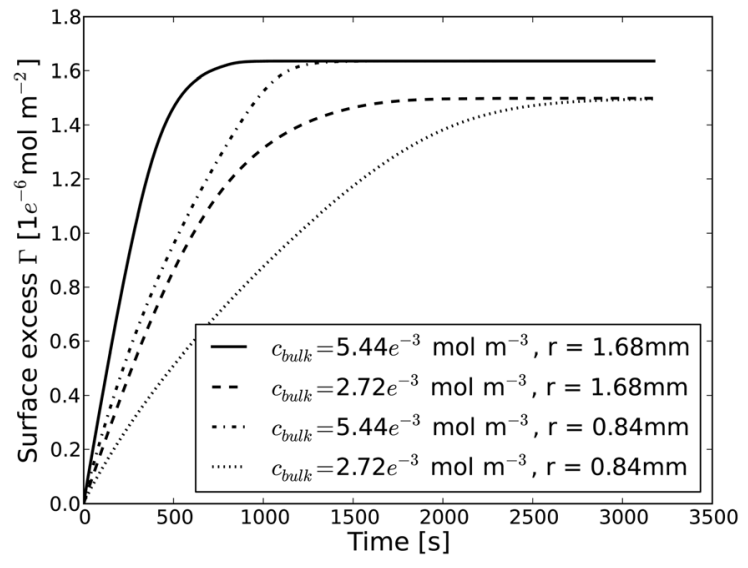

Fig. 3 Surface excess of the droplet for two different radii and bulk concentrations.

Gu. ${ }^{8}$ In Fig. 3, the development of the surface excess is shown for the described radius of the droplet and half of this radius to gain further insight. A lower concentration in the bulk results in a slower increase of the excess and a lower final excess value. The final excess values are reached after approximately $1000 \mathrm{~s}$ for the higher concentration and 2000s for the lower concentration. Those time scales can also be found in the literature, ${ }^{8}$ where also a comparison of the surface excess at the same two concentrations is given. The reason for this long-lasting adsorption process is the low protein concentration that is assumed here.

The droplet with the smaller radius has the same distance between the surface and the bulk region. Therefore, the trend of the surface excess only deviates at the beginning of the adsorption process and comes to the same equilibrium values as for the larger radius.

Fig. 4 shows the evolution of the concentration field starting at the radial position where the bulk concentration ends (dashed line in Fig. 2) and ending at the surface (solid line in Fig. 2). At the very beginning, the concentration in the domain is constantly set to the bulk concentration. Only the surface has

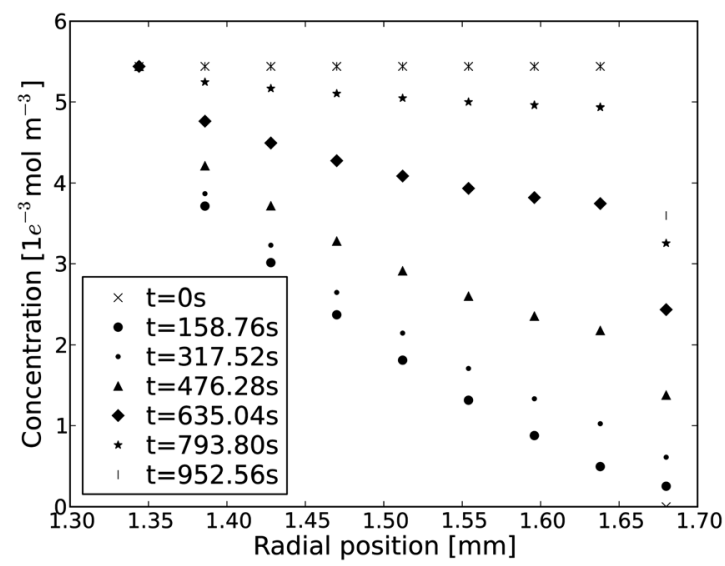

Fig. 4 Concentration distribution in the radial direction for different times during the simulation for $r=1.68 \mathrm{~mm}$ and $c_{\text {bulk }}=5.44 \times 10^{-3}$ $\mathrm{mol} \mathrm{m}{ }^{-3}$. zero concentration since the surface excess is also zero. This introduces a concentration jump from the domain to the surface. In the subsequent time steps, the surface excess increases, and hence the concentration also increases at the surface. The concentration gradient in the domain becomes smaller. The point in the bulk concentration area remains constant at the initial value. At equilibrium, the initial concentration is in the whole domain and at the surface the concentration is slightly lower owing to the equilibrium surface excess there.

Generally, all simulations are without advective effects since a quiescent droplet is investigated. Nevertheless, the relaxation times are such that they allow also advection and hydrodynamics in general.

This model can be applied to different geometries since the general diffusion-advection equation is solved and the flux is computed with the local normal. Considering in addition to the adsorption isotherm also the equation of state gives a relationship between the surface excess and the surface pressure and therefore foam stability. With this local dynamic surface tension, one can gain further insights into the change of flow field in lamellae due to protein adsorption.

The previous validation scenario was made for very low protein concentrations. For realistic protein concentrations in food systems which are two orders of magnitude larger, the influence of the diffusion has to be discussed critically. This is done in the following section.

\section{Diffusive and hydrodynamic time scales}

The diffusion coefficients for proteins in water are very small. Therefore, the investigation of different time scales deserves special attention. Looking at the one-dimensional diffusionadvection eqn (12) in the $x$-direction with velocity $u$

$$
\frac{\partial c}{\partial t}=\frac{\partial}{\partial x}\left(D \frac{\partial}{\partial x} c\right)-\frac{\partial}{\partial x}(c u)
$$

and introducing dimensionless parameters denoted with an asterisk

$$
D^{*}=\frac{D}{D_{\mathrm{c}}} ; c^{*}=\frac{c}{c_{\mathrm{bulk}}} ; x^{*}=\frac{x}{L} ; u^{*}=\frac{u}{u_{\mathrm{c}}} ; t^{*}=\frac{t}{t_{\mathrm{c}}}
$$

where $D_{\mathrm{c}}, c_{\mathrm{bulk}}, L, u_{\mathrm{c}}$ and $t_{\mathrm{c}}$ are characteristic values yields the dimensionless version of (22)

$$
\underbrace{\frac{c_{\text {bulk }}}{t_{\mathrm{c}}}}_{A} \frac{\partial c^{*}}{\partial t^{*}}=\underbrace{\frac{D_{\mathrm{c}} c_{\text {bulk }}}{L^{2}}}_{B} \frac{\partial}{\partial x^{*}}\left(D^{*} \frac{\partial}{\partial x^{*}} c^{*}\right)-\underbrace{\frac{c_{\text {bulk }} u_{\mathrm{c}}}{L}}_{C} \frac{\partial}{\partial x^{*}}\left(c^{*} u^{*}\right) .
$$

Assuming in addition to diffusive effects also advective effects enforces transient scenarios. In food production processes, an example is the development of bubbles at a membrane, or, taking a single bubble for simplicity, a bubble developing at a submerged orifice rises into a protein solution. Realistic quantities for this scenario are listed in Table 1 and determine $A=330 \mathrm{~mol} \mathrm{~s}^{-1} \mathrm{~m}^{-3}, B=2.31 \times 10^{-4} \mathrm{~mol} \mathrm{~s}^{-1} \mathrm{~m}^{-3}$ and $C=26.4 \mathrm{~mol} \mathrm{~s}^{-1} \mathrm{~m}^{-3}$. Since $B$ is five to six orders of 
Table 1 Characteristic quantities for bubble development at a submerged orifice in a protein solution

\begin{tabular}{|c|c|c|}
\hline Quantity & Description & Value \\
\hline$L$ & $\begin{array}{l}\text { Bubble radius (typical bubble size in } \\
\text { BLG foams) }\end{array}$ & $1 \times 10^{-4} \mathrm{~m}$ \\
\hline$u_{\mathrm{c}}$ & $\begin{array}{l}\text { Rising velocity for spherical bubbles } \\
\text { in water and } \text { air }^{30}\end{array}$ & $8 \times 10^{-3} \mathrm{~m} \mathrm{~s}^{-1}$ \\
\hline$t_{\mathrm{c}}$ & $\begin{array}{l}\text { Developing time of a bubble from a } \\
\text { submerged orifice }\end{array}$ & $1 \times 10^{-3} \mathrm{~s}$ \\
\hline$D_{\mathrm{c}}$ & Diffusion coefficient for BLG in water ${ }^{31}$ & $7 \times 10^{-11} \mathrm{~m}^{2} \mathrm{~s}^{-1}$ \\
\hline$c_{\text {bulk }}$ & Typical concentration of BLG in milk ${ }^{1}$ & $0.33 \mathrm{~mol} \mathrm{~m}^{-3}$ \\
\hline
\end{tabular}

magnitude smaller, the diffusive term for the described scenario is negligible. The diffusion process must only be simulated for protein solutions with very low concentrations or static flows where the only change in concentration is due to diffusion. Those scenarios are only of interest for long-term observations as in Section 2.4.2. Highly dynamic processes achieve a change in the concentration field only by advection.

Focusing on the advection process of protein concentrations, it is interesting to estimate the distance between two proteins in a solution with a concentration as in milk. Taking the concentration as in Table 1 and the Avogadro-constant $6 \times 10^{23} \mathrm{~mol}^{-1}$, the number of proteins in a cube with edge length $1 \mu \mathrm{m}$ is $19.6 \times 10^{4}$. Assuming the protein diameter to be $d_{\mathrm{BLG}}=3.5 \mathrm{~nm},{ }^{1}$ the average displacement between two proteins is $13.7 \mathrm{~nm}$. The diffusion velocity of particles can be written as

$$
\frac{\Delta x}{t_{\Delta x}}=\frac{2 D}{\Delta x}
$$

with distance $\Delta x$ and time to diffuse across this distance $t_{\Delta x}{ }^{32}$ With the diffusion coefficient from Table 1 , the time for a protein to travel a distance of $13.7 \mathrm{~nm}$ is

$$
t_{\Delta x}=\frac{\Delta x^{2}}{2 D}=1.34 \mu \mathrm{s} .
$$

This means that within this time, an empty protein position in the solution close to the surface is again occupied by another protein from the solution. At this concentrations, the advection of proteins can also be neglected and the bulk concentration can be assumed everywhere around the surface. This holds for those cases where the other, macroscopic and mesoscopic, processes occur at larger time scales than this diffusive transport time in the microsecond range.

\section{Bubble-bubble interaction}

The previous explanation regarding the influence of diffusion and advection on protein adsorption allows us to assume a global reduced surface tension for protein solutions compared with water for the following scenario. Two bubbles are placed at different distances close to the bottom of a box. Owing to gravity, the bubbles start to rise and deform due to buoyant forces, surface tension and the interaction between them. For the liquid phase, the physical parameters are chosen for water $\left(\rho=1000 \mathrm{~kg} \mathrm{~m}^{-3}\right.$ and $\left.\nu=1 \times 10^{-6} \mathrm{~m}^{2} \mathrm{~s}^{-1}\right)$, the gravity is $g=9.81 \mathrm{~m} \mathrm{~s}^{-2}$ and the diameter of one bubble is around $d_{\text {bubble }}=278 \mu \mathrm{m}$. The surface tension varies between water $\left(\sigma_{\mathrm{w}}=72 \times 10^{-3} \mathrm{~N} \mathrm{~m}^{-1}\right)$ and a $\beta$-casein solution at equilibrium $\left(\sigma_{\mathrm{p}}=50 \times 10^{-3} \mathrm{~N} \mathrm{~m}^{-1}\right)$, as reported by Miller et al. ${ }^{5}$ The simulated time is approximately $25 \mathrm{~ms}$ and the vertical distance that the bubbles travel during this time is around $0.5 \mathrm{~mm}$. Hence for this scenario, the assumption of global and instantaneous change of surface tension is correct according to Section 3. The simulation can help to understand phenomena that occur during foam development, e.g. air injection into the liquid through a membrane. The bubbles produced detach from the membrane due to gravity and rise closely packed. Since this is a very complex scenario with the interaction between bubble growth, deletion and drainage, the subdivision into smaller problems is evident. Also, the interstitial film withdrawal, which causes bubble collapse here, is only one possible mechanism for foam decay.

\subsection{Secondary motions}

To understand the stability of foam lamellae, it is crucial to investigate secondary motions. Secondary motions are the part of the flow field different from the main flow. For the rising bubbles, the main flow is in the direction of the buoyant force. The secondary motions occur in addition to this main flow and the magnitude is significantly smaller. Nevertheless, these small velocities are important since they come from the bubble interaction and indicate the stability of the lamellae between them.

Thinking of two extreme cases regarding secondary motions can help to understand the phenomenon better, which is analyzed with simulations in the following. Two bubbles are rising close to each other with high surface tension, they are fairly stiff, and as soon as they approach each other the liquid is squeezed out (secondary motions) and the lamella ruptures, which results in coalescence. Assuming the same scenario with a very low surface tension means that the bubbles are more deformable, and instead of squeezing out the liquid they change their shape slightly and thus maintain the initial distance and form a stable lamella.

This experiment is examined in the following by simulations.

\subsection{Results}

The simulation domain is discretized by 300 cells in every direction. Bubbles are resolved by 32 cell diameters, which is sufficient. The boundaries of the domain represent no-slip

Table 2 Coalescence between two bubbles due to different initial distances and surface tensions

\begin{tabular}{llc}
\hline & \multicolumn{2}{c}{ Coalescence } \\
\cline { 2 - 3 } Distance & $\sigma_{\mathrm{w}}$ & $\sigma_{\mathrm{p}}$ \\
\hline $43 \mu \mathrm{m}$ & Yes & Yes \\
$52 \mu \mathrm{m}$ & Yes & No \\
$61 \mu \mathrm{m}$ & No & No
\end{tabular}




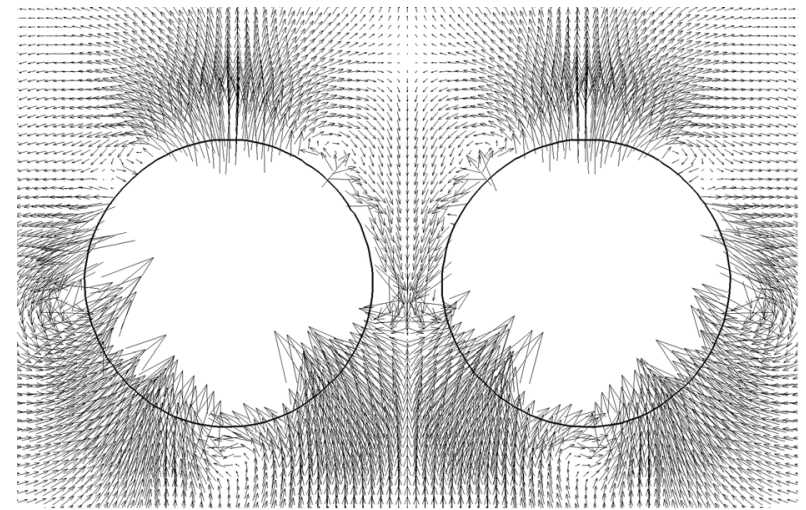

(a) $\sigma_{w}$, after $1.23 \mathrm{~ms}$

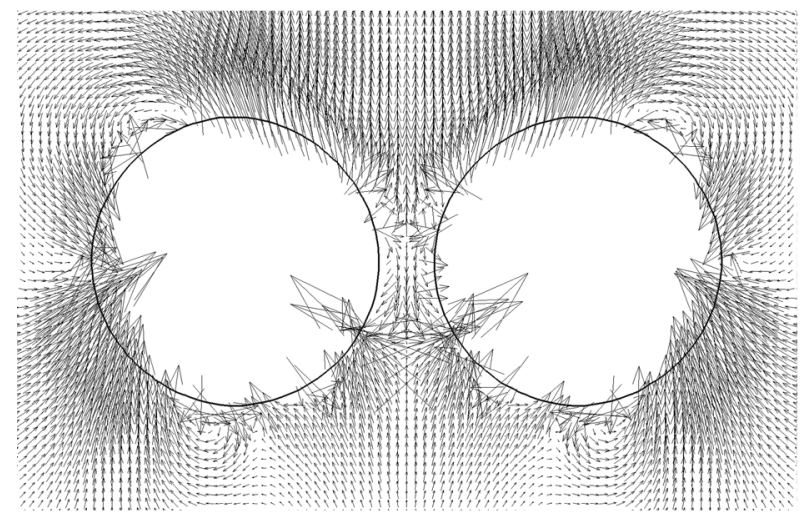

(c) $\sigma_{w}$, after $2.47 \mathrm{~ms}$

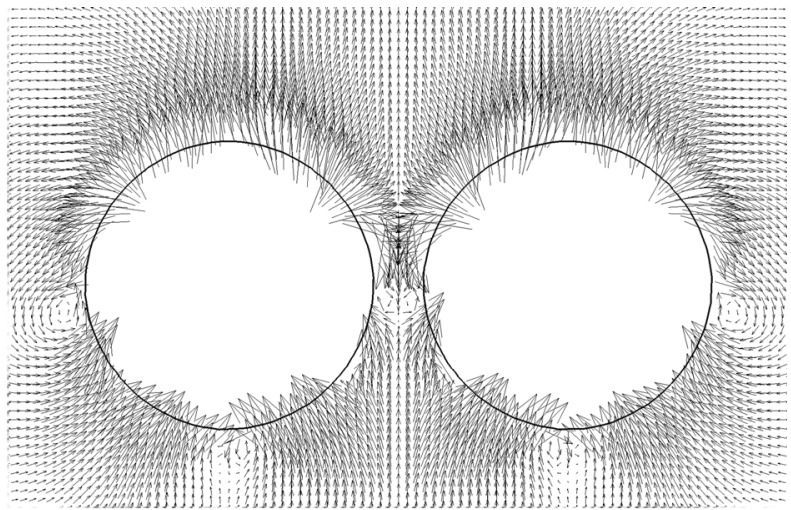

(b) $\sigma_{p}$, after $1.23 \mathrm{~ms}$

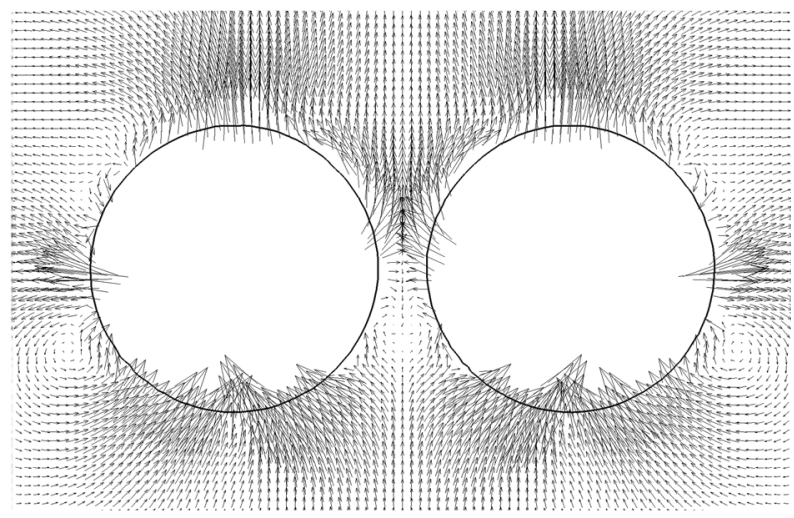

(d) $\sigma_{p}$, after $2.47 \mathrm{~ms}$

Fig. 5 Velocity fields in the plane tangential to gravity, cutting both bubbles through the midpoint.

walls. On that account, the distance of the bubbles from the walls is chosen large enough. The domain is partitioned into 600 blocks of size $50 \times 30 \times 30$ and distributed onto 50 nodes of a cluster. ${ }^{33}$ The computation time for 40000 time steps is approximately 14 hours. In Table 2, coalescence for two surface tensions with different initial distances is summarized. Increasing the distance and decreasing the surface tension lead to less coalescence.

Studying the flow field around the bubbles with distance 52 $\mu \mathrm{m}$, where the bubbles with high surface tension coalesce and those with lower surface tension do not, can explain the results. Fig. 5 shows the mentioned secondary motions at two early time steps before one scenario coalesces. For pure water, the velocity between the bubbles is much higher than for water with proteins. Also, the distance between the two bubbles remains almost the same for the lower surface tension. In the other case, the distance first increases due to stronger repulsion and deformation of the surface and subsequently starts to decrease again, which leads to the final collapse.

These motions of the complete bubbles are visualized in Fig. 6 for all initial distances given in Table 2. It shows the surfaces of the bubbles with evolution of time. Since the bubbles rise, the highest point of the surface basically represents the time progress. For the smallest distance where both scenarios collapse, it can be seen that the bubbles with the higher surface tensions first repel each other and subsequently come together by a contrary movement. At the lower surface tension, this movement is less pronounced but has the same result, a collapse. Regarding the next larger distance, the bubbles with the high surface tension behave almost the same. The other scenario does not show this motion at all at the beginning and only after some time a rejection while rising. This motion is due to the main flow behaviour coming from the surrounding box. It is actually different from an infinite extended domain, and the fluid, which is displaced due to the rising bubbles, has to make its way again to the bottom by developing two vortices with the magnitude of the domain size. At the largest distance, the repulsion remains for both scenarios, but is much larger for the high surface tension case.

\section{Conclusions}

A numerical method is proposed to model the diffusionadvection driven adsorption with the FSLBM. The crucial point here is the correct handling of the boundaries with respect to adsorption and concentration fluxes. Even though difficulties arise due to different time scales for diffusion and hydrodynamics, which are characterized by the diffusion coefficient and the kinematic viscosity, a reasonable set-up is possible. The validation against the literature yielded good results.

Considering realistic protein concentrations as in e.g. milk, the resultant time scales for diffusion and the average distances 


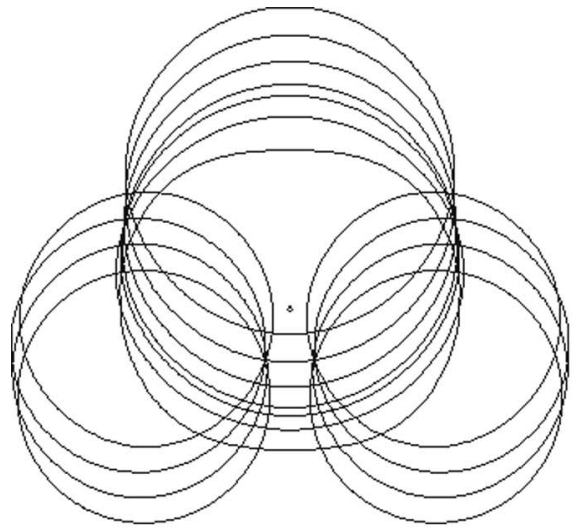

(a) $\sigma_{w}$, initial distance $43 \mu \mathrm{m}$

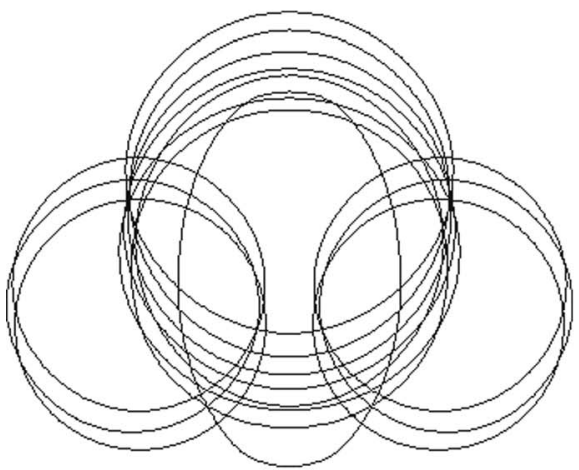

(c) $\sigma_{w}$, initial distance $52 \mu \mathrm{m}$
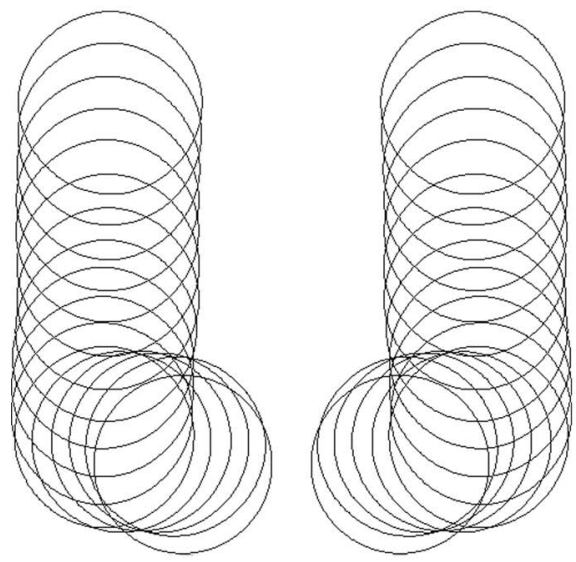

(e) $\sigma_{w}$, initial distance $61 \mu \mathrm{m}$

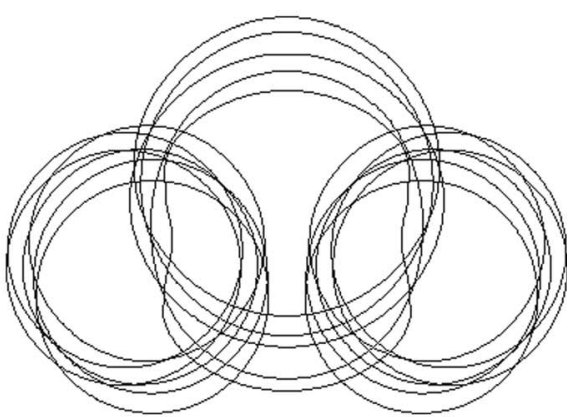

(b) $\sigma_{p}$, initial distance $43 \mu \mathrm{m}$
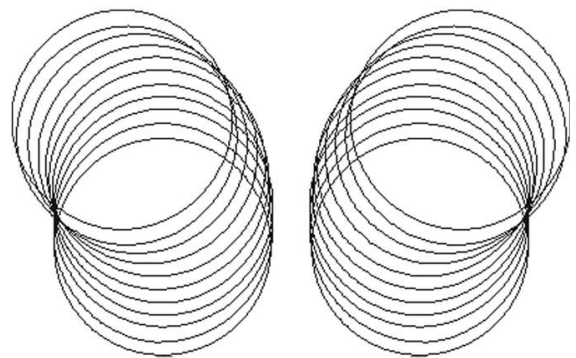

(d) $\sigma_{p}$, initial distance $52 \mu \mathrm{m}$
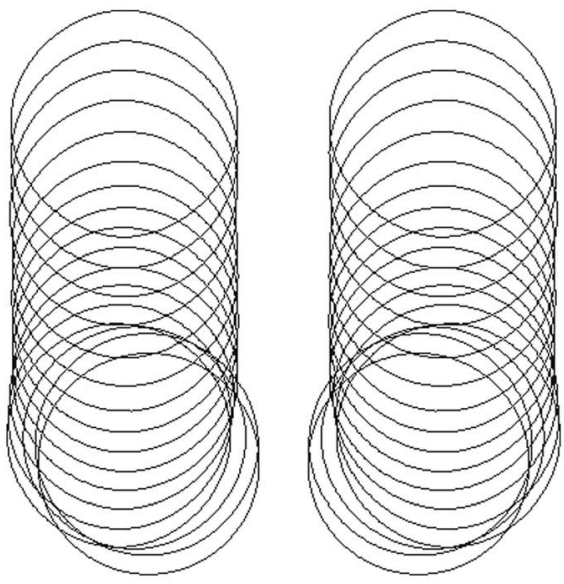

(f) $\sigma_{p}$, initial distance $61 \mu \mathrm{m}$

Fig. 6 Isosurfaces representing the bubbles for sequent time steps.

of proteins in the solution give rise to a simplification. For low concentrations the developed method is needed, but for higher concentrations an instantaneous change of surface tension due to protein adsorption can be assumed. Therefore, the time and space scales for the actual macroscopic scenario must be compared with those of the diffusion and adsorption processes of the proteins.

With this insight, the stability of two rising bubbles was analysed in order to investigate aspects of the foam. The bubble-bubble interaction was simulated for surface tensions as in pure water and as in water containing proteins. The results show that lower surface tension means smaller secondary motions and less coalescence of bubbles. Numerical simulations as a tool to analyse foams also demonstrate the positive influence of proteins on the stability of foams and allow for the investigation of secondary motions.

\section{Acknowledgements}

For the simulations presented in this paper, the waLBerla lattice Boltzmann framework ${ }^{34}$ was used. The authors would like to thank all members of the waLBerla team. This research project 
was supported by the German Ministry of Economics and Technology (via AiF) and the FEI (Forschungskreis der Ernährungsindustrie e.V., Bonn), Project AiF 17125 N.

\section{References}

1 H.-D. Belitz, W. Grosch and P. Schieberle, Lehrbuch der Lebensmittelchemie, Springer, 2001

2 A. Ward and L. Tordai, J. Chem. Phys., 1946, 14, 453.

3 K. J. Mysels, J. Phys. Chem., 1982, 86, 4648-4651.

4 X. Li, R. Shaw, G. M. Evans and P. Stevenson, Comput. Chem. Eng., 2010, 34, 146-153.

5 R. Miller, V. Fainerman, E. Aksenenko, M. Leser and M. Michel, Langmuir, 2004, 20, 771-777.

6 V. Fainerman, E. Lucassen-Reynders and R. Miller, Adv. Colloid Interface Sci., 2003, 106, 237-259.

7 G. Gochev, I. Retzlaff, E. V. Aksenenko, V. B. Fainerman and R. Miller, Colloids Surf., A, 2013, 422, 33-38.

8 C. Yang and Y. Gu, Langmuir, 2004, 20, 2503-2511.

9 M.-W. Yang, Y.-L. Hung, H.-F. Huang and S.-Y. Lin, Colloids Surf., A, 2008, 317, 462-472.

10 D. Wolf-Gladrow, J. Stat. Phys., 1995, 79, 1023-1032.

11 M. A. Hussein and T. Becker, Food Biophys., 2010, 5, 161-176.

12 S. Mack, M. A. Hussein and T. Becker, J. Non-Equilib. Thermodyn., 2011, 36, 311-335.

13 A. M. Sayed, M. Hussein and T. Becker, Biomech. Model. Mechanobiol., 2010, 9, 141-151.

14 X. He, N. Li and B. Goldstein, Mol. Simul., 2000, 25, 145156.

15 D. Wolf-Gladrow, Lattice-Gas Cellular Automata and Lattice Boltzmann Models - An Introduction, Springer, 2005.

16 X. He and L.-S. Luo, Phys. Rev. E: Stat. Phys., Plasmas, Fluids, Relat. Interdiscip. Top., 1997, 56(6), 6811-6817.

17 D. Hänel, Molekulare Gasdynamik, Springer, 2004.

18 S. Harris, An Introduction to the Theory of the Boltzmann Equation, Dover Publications, 1971.

19 P. L. Bhatnagar, E. P. Gross and M. Krook, Phys. Rev., 1954, 94, 511.
20 Y. H. Qian, D. d'Humieres and P. Lallemand, Europhys. Lett., 1992, 17(6), 479-484.

21 M. Junk, A. Klar and L.-S. Luo, J. Comput. Phys., 2005, 210, 676-704.

22 C. Körner, T. Pohl, U. Rüde, N. Thürey and T. Zeiser, Numerical Solution of Partial Differential Equations On Parallel Computers, Lecture Notes In Computational Science And Engineering, Springer, 2006, vol. 51, pp. 439465.

23 T. Pohl, PhD thesis, University of Erlangen-Nuremberg, 2007. 24 S. Donath, C. Feichtinger, T. Pohl, J. Götz and U. Rüde, EuroPar 2009 Parallel Processing, Springer, 2009, vol. 5704, pp. 735-746.

25 D. Anderl, S. Bogner, C. Rauh, U. Rüde and A. Delgado, Comput. Math. Appl., 2014, 67(2), 331-339.

26 E. Attar and C. Körner, Int. J. Heat Fluid Flow, 2011, 32, 156163.

27 M. Junk and D. Kehrwald, ITWM Report, 2006.

28 J. D. Sterling and S. Chen, J. Comput. Phys., 1996, 123, 196206.

29 R. A. Worthing, J. Mozer and G. Seeley, Phys. Rev. E: Stat. Phys., Plasmas, Fluids, Relat. Interdiscip. Top., 1997, 56, 2243.

30 L. Amaya-Bower and T. Lee, Comput. Fluids, 2010, 39, 11911207.

31 H. A. Sober, R. A. Harte and E. K. Sober, Handbook of Biochemistry: Selected Data for Molecular Biology, Chemical Rubber Company, 1970.

$32 \mathrm{Gm}$ bH Wiley Information Services, ChemgaPedia - Jump to Knowledge, http:/www.chemgapedia.de/vsengine/vlu/vsc/ $\mathrm{de} / \mathrm{ch} / 8 / \mathrm{bc} / \mathrm{vlu} /$ transport/pass_transport.vlu/Page/vsc/de/ch/ 8/bc/transport/diffusion1.vscml.html.

33 RRZE Regionales Rechen Zentrum Erlangen, Lima Cluster, http://www.rrze.uni-erlangen.de/dienste/arbeiten-rechnen/ hpc/systeme/lima-cluster.shtml.

34 U. o. E.-N. Lehrstuhl für Informatik 10, WaLBerla - lattice Boltzmann framework, 2006-2013, http:/www10.informatik. uni-erlangen.de/de/Research/Projects/walberla. 